\title{
Explicit characterization of some commuting differential operators of rank 2
}

\section{V.Oganesyan}

\section{Introduction}

If two differential operators

$$
L_{n}=\sum_{i=0}^{n} u_{i}(x) \partial_{x}^{i}, \quad L_{m}=\sum_{i=0}^{m} v_{i}(x) \partial_{x}^{i}
$$

commute, then there is a nonzero polynomial $R(z, w)$ such that $R\left(L_{n}, L_{m}\right)=$ 0 (see [1]). The curve $\Gamma$ defined by $R(z, w)=0$ is called the spectral curve. The genus of the curve $R(z, w)=0$ is called the genus of commuting pair. If

$$
L_{n} \psi=z \psi, \quad L_{m} \psi=w \psi,
$$

then $(z, w) \in \Gamma$. For almost all $(z, w) \in \Gamma$ the dimension of the space of common eigenfunctions $\psi$ is the same. The dimension of the space of common eigenfunctions of two commuting differential operators is called the rank. The rank is a common divisor of $m$ and $n$.

If the rank equals 1 , then there are explicit formulas for coefficients of commutative operators in terms of Riemann theta-functions (see [2]).

The case when rank is greater than one is much more difficult. The first examples of commuting ordinary scalar differential operators of the nontrivial ranks 2 and 3 and the nontrivial genus $\mathrm{g}=1$ were constructed by Dixmier [8] for the nonsingular elliptic spectral curve $w^{2}=z^{3}-\alpha$, where $\alpha$ is an arbitrary nonzero constant:

$$
\begin{gathered}
L=\left(\partial_{x}^{2}+x^{3}+\alpha\right)^{2}+2 x, \\
M=\left(\partial_{x}^{2}+x^{3}+\alpha\right)^{3}+3 x \partial_{x}^{2}+3 \partial_{x}+3 x\left(x^{2}+\alpha\right),
\end{gathered}
$$

where $L$ and $M$ is the commuting pair of the Dixmier operators of rank 2, genus 1 . There is an example

$$
\begin{gathered}
L=\left(\partial_{x}^{3}+x^{2}+\alpha\right)^{2}+2 \partial_{x} \\
M=\left(\partial_{x}^{3}+x^{2}+\alpha\right)^{3}+3 \partial_{x}^{4}+3\left(x^{2}+\alpha\right) \partial_{x}+3 x
\end{gathered}
$$

This work was supported by the program "Leading Scientific Schools" under grant NSh-4833.2014.1. 
where $L$ and $M$ is the commuting pair of the Dixmier operators of rank 3, genus 1 .

The general classification of commuting ordinary differential operators of rank greater than 1 was obtained by Krichever 3]. The general form of commuting operators of rank 2 for an arbitrary elliptic spectral curve was found by Krichever and Novikov [4]. The general form of operators of rank 3 for an arbitrary elliptic spectral curve (the general commuting operators of rank 3, genus 1 are parameterized by two arbitrary functions) was found by Mokhov [5], [6]. Also operators of rank greater than 1 was considered in [15], 16].

Mironov in [7] constructed examples of operators

$$
\begin{gathered}
L=\left(\partial_{x}^{2}+A_{3} x^{3}+A_{2} x^{2}+A_{1} x+A_{0}\right)^{2}+g(g+1) A_{3} x, \\
M^{2}=L^{2 g+1}+a_{2 g} L^{2 g}+\ldots+a_{1} L+a_{0},
\end{gathered}
$$

where $a_{i}$ are some constants and $A_{i}, A_{3} \neq 0$, are arbitrary constants. Operators $L$ and $M$ are commuting operators of rank 2, genus g. Furthermore, in [9] it was proved by Mironov that operators $L_{1}$ and $M_{1}$

$$
\begin{gathered}
L_{1}=\left(\partial_{x}^{2}+\alpha_{1} \mathcal{P}(x)+\alpha_{0}\right)^{2}+\alpha_{1} g_{2} g(g+1) \mathcal{P}(x), \quad \alpha_{1} \neq 0, \\
M_{1}^{2}=L_{1}^{2 g+1}+a_{2 g} L_{1}^{2 g}+\ldots+a_{1} L_{1}+a_{0},
\end{gathered}
$$

where $a_{i}$ are some constants, $\alpha_{i}$ are arbitrary constants and $\mathcal{P}$ satisfies the equation

$$
\left(\mathcal{P}^{\prime}(x)\right)^{2}=g_{2} \mathcal{P}^{2}(x)+g_{1} \mathcal{P}(x)+g_{0}, \quad g_{2} \neq 0,
$$

where $g_{1}$ and $g_{2}$ are arbitrary constants, is a commuting pair of rank 2 , genus $g$.

Let $\wp(x)$ be the Weierstrass elliptic function satisfying the equation $\left(\wp^{\prime}(x)\right)^{2}=$ $4 \wp^{3}(x)+g_{2} \wp(x)+g_{3}$. Mironov proved in [9] that operators $L_{2}$ and $M_{2}$

$$
\begin{gathered}
L_{2}=\left(\partial_{x}^{2}+\alpha_{1} \wp(x)+\alpha_{0}\right)^{2}+s_{1} \wp(x)+s_{2} \wp^{2}(x), \\
M_{2}^{2}=L_{2}^{2 g+1}+b_{2 g} L_{2}^{2 g}+\ldots+b_{1} L_{2}+b_{0},
\end{gathered}
$$

where $b_{i}$ are some constants, $\alpha_{1}=\frac{1}{4}-2 g^{2}-2 g, s_{1}=\frac{1}{4} g(g+1)\left(16 \alpha_{0}+5 g_{2}\right)$, $s_{2}=-4 g(g+2)\left(g^{2}-1\right)$ and $\alpha_{0}$ is an arbitrary constant, are also a commuting pair of rank 2, genus $g$. Using the same methods many other examples were found [17], [18].

Examples of commuting ordinary differential operators of arbitrary genus and arbitrary rank with polynomial coefficients were constructed in [11] by Mokhov.

Consider

$$
\begin{gathered}
L_{1}=\left(\partial_{x}^{2}+A_{6} x^{6}+A_{2} x^{2}\right)^{2}+16 g(g+1) A_{6} x^{4}, \\
L_{2}=\left(\partial_{x}^{2}+A_{4} x^{4}+A_{2} x^{2}+A_{0}\right)^{2}+4 g(g+1) A_{4} x^{2},
\end{gathered}
$$


where $g \in \mathbb{N}, A_{6} \neq 0, A_{4} \neq 0, A_{2}, A_{0}$ are arbitrary constants. Operators $L_{1}$ and $L_{2}$ commute with operators of order $4 \mathrm{~g}+2$ (see [12], [13]). The spectral curves of these operators have the form $w^{2}=z^{2 m+1}+a_{2 m} z^{2 m}+\ldots+a_{1} z+a_{0}$. Moreover, the following theorems are proved in [12], [13].

1) If $L=\left(\partial_{x}^{2}+A_{n} x^{n}+A_{n-1} x^{n-1}+\ldots+A_{0}\right)^{2}+B_{k} x^{k}+B_{k-1} x^{k-1}+\ldots+B_{0}$, where $n>3, n \in \mathbb{N}, A_{n} \neq 0, B_{k} \neq 0$, commutes with a differential operator $\mathrm{M}$ of order $4 g+2$ and $\mathrm{M}, \mathrm{L}$ are operators of rank 2, then $k=n-2$ and $B_{k}=(n-2)^{2} m(m+1) A_{n}$ for some $m \in \mathbb{N}$.

2) For $n>6$ operator $L=\left(\partial_{x}^{2}+A_{n} x^{n}\right)^{2}+B_{n-2} x^{n-2}$ does not commute with any differential operator $M$ of order $4 g+2$, where $M$ and $L$ could be a pair of rank 2 .

3) If $n=5$, then $L=\left(\partial_{x}^{2}+A x^{5}\right)^{2}+18 A x^{3}, A \neq 0$, commutes with a differential operator $\mathrm{M}$ of order $4 g+2$ for all $g$ and M,L are operators of rank 2 .

4) The operator $\left(\partial_{x}^{2}+A x^{5}\right)^{2}+9 m(m+1) A x^{3}, A \neq 0$ does not commute with any differential operator $\mathrm{M}$ of order $4 g+2$, for $m>1$, where $\mathrm{M}$ and $\mathrm{L}$ could be a pair of rank 2 .

Let us consider the operator

$$
L_{4}=\partial_{x}^{4}+u(x)
$$

Assume that $u(x)$ has poles at points $a_{1}, a_{2}, \ldots$ In a neighborhood of $a_{i}$

$$
u(x)=\frac{\varphi_{i,-k}}{\left(x-a_{i}\right)^{k}}+\frac{\varphi_{i,-k+1}}{\left(x-a_{i}\right)^{k-1}}+\ldots+\varphi_{i, 0}+\varphi_{i, 1}\left(x-a_{i}\right)+O\left(\left(x-a_{i}\right)^{2}\right) .
$$

The main results of this paper are the following.

\section{Theorem 1.1}

If $L_{4}=\partial_{x}^{4}+u(x)$ commutes with a differential operator $M$ of order $4 g+2$ and $M, L_{4}$ are operators of rank 2, then $u(x)$ can have pole only of order 4 , $\varphi_{i,-4}=n_{i}\left(4 n_{i}+1\right)\left(4 n_{i}+3\right)\left(4 n_{i}+4\right), n_{i} \in \mathbb{N}, \varphi_{i, 4 k-l}=0$, where $k=0, \ldots, n_{i}$, $l=1,2,3$. Moreover $\varphi_{i, 4 r-1}=\varphi_{i, 4 r-3}=0$, where $r=n_{i}+1, \ldots, g$. Function $u(x)$ can't have isolated pole at infinity. 


\section{Corollary 1.2}

Suppose $u(x)$ is elliptic, simply-periodic or rational function and hasn't isolated singularity at infinity. Let $a_{1}$ be the unique pole in a fundamental parallelogram, a period-strip or the complex plane respectively. The operator $L_{4}=\partial_{x}^{4}+u(x)$ commutes with an operator $M$ of order $4 g+2$ and $M, L_{4}$ are operators of rank 2 if and only if $\varphi_{1,-4}=g(4 g+1)(4 g+3)(4 g+4)$, $\varphi_{1,4 k-l}=0$, where $k=0, \ldots, g, l=1,2,3$.

\section{Corollary 1.3}

Let $\wp(x)$ be the Weierstrass elliptic function satisfying the equation $\left(\wp^{\prime}(x)\right)^{2}=$ $4 \wp^{3}(x)+g_{2} \wp(x)+g_{3}$. The Operator

$$
L_{4}=\partial_{x}^{4}+n(4 n+1)(4 n+3)(4 n+4) \wp^{2}(x),
$$

where $n \in \mathbb{N}$, commutes with an operator of order $4 n+2$ if and only if $\wp(x)$ is a solution of the equation $\left(\wp^{\prime}(x)\right)^{2}=4(\wp(x))^{3}+g_{2} \wp(x)$. If $g_{2}=0$, then we obtain $L_{4}=\partial_{x}^{4}+\frac{n(4 n+1)(4 n+3)(4 n+4)}{x^{4}}$. Calculations show that for $n$ less than 8 the spectral curve is non-singular for almost all $g_{2}$.

\section{Example 1.4}

Let $\wp(x)$ be the Weierstrass elliptic function satisfying the equation $\left(\wp^{\prime}(x)\right)^{2}=$ $4 \wp^{3}(x)+g_{2} \wp(x)+g_{3}$. Consider the operator

$$
L_{4}=\partial_{x}^{4}+280 \wp^{2}(x)+280 \wp^{2}(x-a),
$$

Suppose that $g_{3}=0$ and $a=\omega_{1}, \omega_{2}$ or $\omega_{1}+\omega_{2}$, where $\omega_{i}$ are half-periods. Then $L_{4}$ commutes with an operator of order 6 .

Analyzing the proof of Theorem 1 we obtain the following conjecture

\section{Conjecture}

Let $u(x)$ be elliptic (meromorphic) function with finite number of poles in a fundamental parallelogram ( in $\mathbb{C}$ and hasn't isolated pole at infinity). Assume $S=\sum_{i=1}^{i=m} n_{i}+1$, where $m$ is number of poles. If $\varphi_{i,-4}=n_{i}\left(4 n_{i}+\right.$ 1) $\left(4 n_{i}+3\right)\left(4 n_{i}+4\right), \varphi_{i, 4 k-l}=0, \varphi_{i, 4 r-1}=\varphi_{i, 4 r-3}=0$, where $k=0, \ldots, n_{i}$, $r=n_{i}+1, \ldots, S$ and $l=1,2,3$, then $L_{4}=\partial_{x}^{4}+u(x)$ commutes with a differential operator of order $4 S+2$.

\section{Theorem 1.5}

If $L_{4}$ commutes with a differential operator $M$ of order $4 g+2, M$ and $L_{4}$ are operators of rank 2 and $u(x)$ has pole at $a_{i}$, then solutions of the equation $\psi^{(4)}(x)+u(x) \psi(x)=\lambda \psi(x)$ have singularities at $a_{i}$ of the following type 
$x^{\sigma_{i, r}} g(x)$, where $r=1,2,3,4$ and $g(x)$ is holomorphic at $a_{i}$,

$\sigma_{i, 1}=\frac{1}{2}\left(1-4 n_{i}-\sqrt{1-16 n_{i}-16 n_{i}^{2}}\right)$

$\sigma_{i, 2}=\frac{1}{2}\left(1-4 n_{i}+\sqrt{1-16 n_{i}-16 n_{i}^{2}}\right)$

$\sigma_{i, 3}=\frac{1}{2}\left(5+4 n_{i}-\sqrt{1-16 n_{i}-16 n_{i}^{2}}\right)$

$\sigma_{i, 4}=\frac{1}{2}\left(5+4 n_{i}+\sqrt{1-16 n_{i}-16 n_{i}^{2}}\right)$.

This means that common eigenfunctions of commuting operators always have branch points. Hence $L_{4}$ doesn't commute with an operator of odd order.

The author wishes to express gratitude to Professor O. I. Mokhov for advice and help in writing this paper.

\section{Commuting differential operators of rank 2}

Consider the operator

$$
L=\left(\partial_{x}^{2}+V(x)\right)^{2}+W(x)
$$

From [7] it follows that the operator $L$ commutes with an operator $M$ of order $4 g+2$ and the spectral curve of $L$ and $M$ is hyperelliptic curve of genus $g$ and hence operators $L$ and $M$ are operators of rank 2, if and only if there exists a polynomial

$$
Q=z^{g}+a_{1}(x) z^{g-1}+a_{2}(x) z^{g-2}+\ldots+a_{g-1}(x) z+a_{g}(x)
$$

that the following relation is satisfied

$$
Q^{(5)}+4 V Q^{\prime \prime \prime}+6 V^{\prime} Q^{\prime \prime}+2 Q^{\prime}\left(2 z-2 W+V^{\prime \prime}\right)-2 Q W^{\prime} \equiv 0,
$$

$Q^{\prime}$ means $\partial_{x} Q$. The spectral curve has the form

$4 w^{2}=4 F(z)=4(z-W) Q^{2}-4 V\left(Q^{\prime}\right)^{2}+\left(Q^{\prime \prime}\right)^{2}-2 Q^{\prime} Q^{\prime \prime \prime}+2 Q\left(2 V^{\prime} Q^{\prime}+4 V Q^{\prime \prime}+Q^{(4)}\right)$.

If $V(x) \equiv 0$, then we have

$$
\begin{gathered}
Q^{(5)}+2 Q^{\prime}(2 z-2 W)-2 Q W^{\prime} \equiv 0 \\
4 w^{2}=4 F(z)=4 z Q^{2}+\left(Q^{\prime \prime}\right)^{2}-2 Q^{\prime} Q^{\prime \prime \prime}+2 Q Q^{(4)} .
\end{gathered}
$$

Using (4) we get

$$
4 Q^{\prime} z \equiv-Q^{(5)}+2 Q W^{\prime}+4 Q^{\prime} W
$$


So we have the following system :

$$
\left\{\begin{array}{l}
a_{1}=W / 2+C_{1} \\
4 a_{2}^{\prime}=-a_{1}^{(5)}+2 a_{1} W^{\prime}+4 a_{1}^{\prime} W \\
\cdots \\
4 a_{i+1}^{\prime}=-a_{i}^{(5)}+2 a_{i} W^{\prime}+4 a_{i}^{\prime} W \\
\cdots \\
4 a_{g}^{\prime}=-a_{g-1}^{(5)}+2 a_{g-1} W^{\prime}+4 a_{g-1}^{\prime} W \\
0=-a_{g}^{(5)}+2 a_{g} W^{\prime}+4 a_{g}^{\prime} W
\end{array}\right.
$$

It is clear from the system that the result in [7] can be formulated in the following way. Let $a_{1}=W / 2+C_{1}$, where $C_{1}$ is an arbitrary constant. Define $a_{i}$ by recursion

$$
a_{i+1}=C_{i+1}+\frac{1}{4} \int\left(-a_{i}^{(5)}+2 a_{i} W^{\prime}+4 a_{i}^{\prime} W\right) d x .
$$

We see that if $V(x) \equiv 0$, then (1) commutes with an operator of order $4 g+2$ and these operators are operators of rank 2 if and only if $a_{g+1} \equiv$ const.

From the proof of Theorem 2 in [7] easy to see that $a_{i}(x)$ is a polynomial in $W(x), W^{\prime}(x), W^{\prime \prime}(x), \ldots$ But we will prove this fact by direct calculations. We have

$$
\begin{gathered}
\int\left(W(x) a_{1}^{\prime}(x)\right) d x=\int\left(\frac{W(x) W^{\prime}(x)}{2}\right)=\frac{W^{2}(x)}{4}, \\
\int\left(3 W^{2}(x) a_{1}^{\prime}(x)-a_{1}^{(5)}(x) W(x)\right) d x= \\
=\frac{1}{2} W^{3}(x)-\frac{1}{4}\left(W^{\prime \prime}(x)\right)^{2}+\frac{1}{2} W^{\prime}(x) W^{\prime \prime \prime}(x)-\frac{1}{2} W(x) W^{(4)}(x) .
\end{gathered}
$$

Let us suppose that $a_{k}, \int W(x) a_{k}^{\prime}(x) d x$ and $\int\left(3 W^{2}(x) a_{k}^{\prime}(x)-a_{k}^{(5)}(x) W(x)\right) d x$ are polynomials in $W(x), W^{\prime}(x), W^{\prime \prime}(x), \ldots$ We obtain

$$
\begin{aligned}
& a_{k+1}(x)=C_{k+1}+\frac{1}{4} \int\left(-a_{k}^{(5)}(x)+2 a_{k}(x) W^{\prime}(x)+4 a_{k}^{\prime}(x) W(x)\right) d x= \\
= & C_{k+1}-\frac{1}{4} \int a_{k}^{(5)}(x) d x+\frac{1}{2} \int \frac{d\left(a_{k}(x) W(x)\right)}{d x} d x+\frac{1}{2} \int a_{k}^{\prime}(x) W(x) d x .
\end{aligned}
$$

So, we see that $a_{k+1}(x)$ is polynomial in $W(x), W^{\prime}(x), W^{\prime \prime}(x) \ldots$ And analogously easy to check that $\int W(x) a_{k+1}^{\prime}(x) d x$ and $\int\left(3 W^{2}(x) a_{k+1}^{\prime}(x)-a_{k+1}^{(5)}(x) W(x)\right) d x$ are polynomial in $W(x), W^{\prime}(x), W^{\prime \prime}(x) \ldots$

Let $L_{4}$ be the operator

$$
L_{4}=\partial_{x}^{4}+u(x)
$$


The commutativity condition of $L_{4}$ and $L_{6}$ is equivalent to the equation

$$
4 C_{1} u^{\prime}(x)+6 u(x) u^{\prime}(x)-u^{(5)}(x)=0,
$$

for some constant $C_{1}$. Consider differential equations in $u(x)$

$$
0=f_{j+1}(x)=C_{i+1}+\int\left(u(x) f_{j}^{\prime}(x)+\frac{u^{\prime}(x) f_{j}(x)}{2}-\frac{f_{j}^{(5)}(x)}{4}\right) d x
$$

where

$$
f_{1}=C_{1}+\frac{u(x)}{2}
$$

We see that $L_{4}$ commutes with an operator of order $4 g+2$ if and only if there exist constants $C_{1}, \ldots, C_{g}$ such that $f_{g+1} \equiv$ const.

\section{Proof of Theorem 1.1 and corollaries}

\subsection{Proof of theorem 1.1}

Assume that $u(x)$ has poles at points $a_{1}, a_{2}, \ldots$ in $\mathbb{C}$.

\section{Lemma 3.1}

If $L_{4}$ commutes with an operator of order $4 g+2$, then $u(x)$ can have poles in $\mathbb{C}$ only of order 4 .

Proof. Assume that $u(x)$ has pole of order $k$ at point $a_{i}$. Since $L_{4}$ commutes with an operator of order $4 g+2$, we have $f_{g+1}^{\prime} \equiv 0$. Suppose $f_{g}$ has pole at point $a_{i}$ of order $m$; then $u(x) f_{g}^{\prime}(x)+\frac{u^{\prime}(x) f_{g}(x)}{2}$ has pole of order $k+m+1$ and $f_{g}^{(5)}(x)$ has pole of order $m+5$. Hence, we see that if $f_{g+1} \equiv 0$, then $\mathrm{k}+\mathrm{m}+1=\mathrm{m}+5$. We get $k=4$.

By $A_{i, m}^{k}$ denote a coefficient in the term $\left(x-a_{i}\right)^{m}$ in Laurent series of $f_{k}$ at point $a_{i}$. We see from Lemma 3.1 that $f_{k}$ has pole of order $4 k$ at point $a_{i}$. Assume that Laurent series of $u(x)$ has the form

$$
\begin{gathered}
u(x)=\frac{\varphi_{i,-4}}{\left(x-a_{i}\right)^{4}}+\frac{\varphi_{i,-3}}{\left(x-a_{i}\right)^{3}}+\frac{\varphi_{i,-2}}{\left(x-a_{i}\right)^{2}}+\frac{\varphi_{i,-1}}{x-a_{i}}+ \\
+\varphi_{i, 0}+\varphi_{i, 1}\left(x-a_{i}\right)+\varphi_{i, 2}\left(x-a_{i}\right)^{2}+\varphi_{i, 3}\left(x-a_{i}\right)^{3}+\varphi_{i, 4}\left(x-a_{i}\right)^{4}++O\left(\left(x-a_{i}\right)^{5}\right) .
\end{gathered}
$$




\section{Lemma 3.2}

If $L_{4}$ commutes with an operator of order $4 g+2$, then $\varphi_{i,-4}=n_{i}\left(4 n_{i}+1\right)\left(4 n_{i}+3\right)\left(4 n_{i}+4\right)$, where $n_{i} \in \mathbb{N}$ and

$$
A_{i,-4 k-4}^{k+1}=\frac{(2 k+1) A_{i,-4 k}^{k}\left(\varphi_{-4}-k(4 k+1)(4 k+3)(4 k+4)\right)}{2 k+2} .
$$

Proof. In a neighborhood of $a_{i}$

$$
f_{j}=\frac{A_{i,-4 j}^{j}}{\left(x-a_{i}\right)^{4 j}}+O\left(\left(x-a_{i}\right)^{-4 j+1}\right) .
$$

Easy to see that

$$
\begin{gathered}
f_{j+1}^{\prime}=\left(\frac{\varphi_{i,-4}}{\left(x-a_{i}\right)^{4}}+O\left(\frac{1}{\left(x-a_{i}\right)^{3}}\right)\right)\left(-\frac{4 j A_{i,-4 j}^{j}}{\left(x-a_{i}\right)^{4 j+1}}+O\left(\left(x-a_{i}\right)^{-4 j}\right)+\right. \\
+\left(-\frac{2 \varphi_{i,-4}}{\left(x-a_{i}\right)^{5}}+O\left(\frac{1}{\left(x-a_{i}\right)^{4}}\right)\right)\left(\frac{A_{i,-4 j}^{j}}{\left(x-a_{i}\right)^{4 j}}+O\left(\left(x-a_{i}\right)^{-4 j+1}\right)+\right. \\
+\frac{j(4 j+1)(4 j+2)(4 j+3)(4 j+4) A_{i,-4 j}^{j}}{\left(x-a_{i}\right)^{4 j+5}}+O\left(\left(x-a_{i}\right)^{-4 j-4}\right)= \\
=-\frac{(4 j+2)\left(\varphi_{i,-4}-j(4 j+1)(4 j+3)(4 j+4)\right) A_{i,-4 j}^{j}}{\left(x-a_{i}\right)^{4 j+5}}+O\left(\left(x-a_{i}\right)^{-4 j-4}\right) .
\end{gathered}
$$

So, integrating we obtain that

$$
A_{i,-4 j-4}^{j+1}=\frac{(2 j+1)\left(\varphi_{i,-4}-j(4 j+1)(4 j+3)(4 j+4)\right) A_{i,-4 j}^{j}}{2 j+2}
$$

If $f_{g+1}^{\prime} \equiv 0$, then the leading term of principal part must vanish. Hence, $\varphi_{i,-4}=n_{i}\left(4 n_{i}+1\right)\left(4 n_{i}+3\right)\left(4 n_{i}+4\right)$ for some $n_{i}$.

\section{Lemma 3.3}

Suppose $j<n_{i}+1$ and in a neighborhood of $a_{i}$

$$
f_{j}=\frac{A_{i,-4 j}^{j}}{\left(x-a_{i}\right)^{4 j}}+O\left(\left(x-a_{i}\right)^{-4 j+1}\right) ;
$$

then $A_{i,-4 j}^{j}>0$ and if $j \geqslant n_{i}+1$, then $A_{i,-4 j}^{j}=0$. 
Proof. Since $\varphi_{i,-4}=n_{i}\left(4 n_{i}+1\right)\left(4 n_{i}+3\right)\left(4 n_{i}+4\right)$ and

$$
A_{i,-4 j-4}^{j+1}=\frac{(2 j+1)\left(\varphi_{i,-4}-j(4 j+1)(4 j+3)(4 j+4)\right) A_{i, 4 j}^{j}}{2 j+2},
$$

we see that if $j<n_{i}+1$, then $A_{i,-4 j}^{j}>0$.

If $j \geqslant n_{i}+1$, then $A_{i,-4 j}^{j}=0$.

Let us prove that $u(x)$ can't have isolated pole at infinity. Assume the converse. Then $u(x)$ has pole at infinity of order $m$. We have

$$
f_{1}=A_{\infty, m}^{1} x^{m}+O\left(x^{m-1}\right)=\frac{\varphi_{\infty, m}}{2}+O\left(x^{m-1}\right)
$$

But $f_{2}$ has pole at infinity of order $2 m$ and $A_{\infty, 2 m}^{2}=\frac{2 m A_{\infty, m}^{1} \varphi_{\infty, m}+m \varphi_{\infty, m} A_{\infty, m}^{1}}{4 m} \neq$ 0 . In general $A_{\infty,(k+1) m}^{k+1}=\frac{2 k m A_{\infty, k m}^{k} \varphi_{\infty, m}+m \varphi_{\infty, m} A_{\infty, k m}^{k}}{2 m(k+1)}=\frac{\varphi_{\infty, m} A_{\infty, m k}^{k}(2 k+1)}{2(k+1)} \neq 0$. So, order of pole increases and there is no $k$ such that $f_{k}$ vanishes.

\section{Lemma 3.4}

If $L_{4}$ commutes with an operator of order $4 g+2$, then $\varphi_{i,-3}=\varphi_{i,-2}=\varphi_{i,-1}=0$ for all $i$.

Proof. By definition, $f_{1}(x)=C_{1}+\frac{u(x)}{2}$ in a neighborhood of $a_{i}$ has the form

$$
f_{1}=\frac{\varphi_{i,-4}}{2\left(x-a_{i}\right)^{4}}+\frac{\varphi_{i,-l}}{2\left(x-a_{i}\right)^{l}}+O\left(\left(x-a_{i}\right)^{-l+1}\right)
$$

where $l=1,2,3$. Therefore, $f_{2}^{\prime}(x)=u(x) f_{1}^{\prime}(x)+\frac{u^{\prime}(x) f_{1}(x)}{2}-\frac{f_{1}^{(5)}(x)}{4}$ in a neighborhood of $a_{i}$

$$
\begin{aligned}
& \left(\frac{\varphi_{i,-4}}{\left(x-a_{i}\right)^{4}}+\frac{\varphi_{i,-l}}{\left(x-a_{i}\right)^{l}}+O\left(\left(x-a_{i}\right)^{-l+1}\right)\right)\left(-\frac{2 \varphi_{i,-4}}{\left(x-a_{i}\right)^{5}}-\frac{l \varphi_{i,-l}}{2\left(x-a_{i}\right)^{l+1}}+O\left(\left(x-a_{i}\right)^{-l}\right)\right)+ \\
& +\left(-\frac{\varphi_{i,-4}}{\left(x-a_{i}\right)^{5}}-\frac{l \varphi_{i,-l}}{4\left(x-a_{i}\right)^{l+1}}+O\left(\left(x-a_{i}\right)^{-l}\right)\right)\left(\frac{\varphi_{i,-4}}{\left(x-a_{i}\right)^{4}}+\frac{\varphi_{i,-l}}{\left(x-a_{i}\right)^{l}}+O\left(\left(x-a_{i}\right)^{-l+1}\right)\right)+ \\
& \quad+\frac{840 \varphi_{i,-4}}{\left(x-a_{i}\right)^{9}}+\frac{l(l+1)(l+2)(l+3)(l+4) \varphi_{i,-l}}{8\left(x-a_{i}\right)^{l+5}}+O\left(\left(x-a_{i}\right)^{-l-4}\right)= \\
& =-\frac{3 \varphi_{i,-4}\left(\varphi_{i,-4}-280\right)}{\left(x-a_{i}\right)^{9}}-\frac{\varphi_{i,-l}(4+l)\left(6 \varphi_{i,-4}-l(l+1)(l+2)(l+3)\right)}{8\left(x-a_{i}\right)^{l+5}}+O\left(\left(x-a_{i}\right)^{-l-4}\right)
\end{aligned}
$$

Integrating, we obtain

$f_{2}(x)=\frac{3 \varphi_{i,-4}\left(\varphi_{i,-4}-280\right)}{8\left(x-a_{i}\right)^{8}}+\frac{\varphi_{i,-l}\left(6 \varphi_{i,-4}-l(l+1)(l+2)(l+3)\right)}{8\left(x-a_{i}\right)^{l+4}}+O\left(\left(x-a_{i}\right)^{-l-3}\right)$ 
Note that $6 \varphi_{i,-4}-l(l+1)(l+2)(l+3)>0$. Consider $f_{k}$, where $k \leqslant n_{i}$.

$$
f_{k}=\frac{A_{i,-4 k}^{k}}{\left(x-a_{i}\right)^{4 k}}+\frac{A_{i,-4 k+4-l}^{k}}{\left(x-a_{i}\right)^{4 k-4+l}}+O\left(\left(x-a_{i}\right)^{-4 k+3-l}\right) .
$$

Let us prove that

$$
A_{i,-4 k-l}^{k+1}=\varphi_{i,-l} K_{i,-4 k-l}^{k+1} \quad \text { for } \quad k \leqslant n_{i},
$$

where $K_{i,-4 k-l}^{k+1}>0$ and doesn't depend on $\varphi_{i,-l}$. The proof is by induction on $k$. We checked this for $k=2$. By the induction hypothesis, $A_{i,-4 k+4-l}^{k}=$ $\varphi_{i,-l} K_{i,-4 k+4-l}^{k}$ and $K_{i,-4 k+4+l}^{k}>0$.

Easy to see that

$$
\begin{gathered}
f_{k+1}=\frac{(2 k+1)\left(\varphi_{i,-4}-k(4 k+1)(4 k+3)(4 k+4)\right) A_{i,-4 k}^{k}}{(2 k+2)\left(x-a_{i}\right)^{4 k+4}}+ \\
+\frac{\varphi_{i,-l} A_{i,-4 k}^{k}(8 k+l)}{2(4 k+l)\left(x-a_{i}\right)^{4 k+l}}+ \\
\frac{(4 k-2+l)\left(4 \varphi_{i,-4}-(4 k-4+l)(4 k-3+l)(4 k-1+l)(4 k+l)\right) A_{i,-4 k+4-l}^{k}}{4(4 k+l)\left(x-a_{i}\right)^{4 k+l}}+\ldots \\
=\frac{A_{i,-4 k-4}^{k+1}}{\left(x-a_{i}\right)^{4 k+4}}+\frac{A_{i,-4 k-l}^{k+1}}{\left(x-a_{i}\right)^{4 k+l}}+O\left(\left(x-a_{i}\right)^{-4 k+1-l}\right)
\end{gathered}
$$

We see that $A_{i,-4 k-l}^{k+1}=\varphi_{i,-l} K_{i,-4 k-l}^{k+1}$. We obtain that $K_{i,-4 k-l}^{k+1}>0$ because $A_{i,-4 k}^{k}>0$, where $k \leqslant n_{i}$. So, (8) is proved.

We know from Lemma 3.2 that $A_{i,-4 n_{i}-4}^{n_{i}+1}=0$. If $k \geqslant n_{i}+1$, then

$$
A_{i,-4 k-l}^{k+1}=\frac{A_{i,-4 k+4-l}^{k}(4 k-2+l)\left(4 \varphi_{i,-4}-(4 k-4+l)(4 k-3+l)(4 k-1+l)(4 k+l)\right)}{4(4 k+l)} .
$$

Easy to see that if $k \geqslant n_{i}+1$, then

$$
\left.4 n_{i}\left(4 n_{i}+1\right)\left(4 n_{i}+3\right)\left(4 n_{i}+4\right)-(4 k-4+l)(4 k-3+l)(4 k-1+l)(4 k+l)\right)<0 .
$$

Finally we obtain that if there exists a $g$ such that $f_{g+1} \equiv 0$, then $A_{i,-4 k+4-l}^{k}=$ $\varphi_{i,-l} K_{i,-4 k+4-l}^{k}=0$ for some $k$. But $K_{i,-4 k+4-l}^{k} \neq 0$ for all $k$. Hence, $\varphi_{i,-l}=$ 0 .

Now let us prove the main part of Theorem 1.1. 
From Lemma 3.4 we know that $f_{1}(x)=C_{1}+\frac{u(x)}{2}$ in a neighborhood of $a_{i}$ has the form

$$
f_{1}=\frac{\varphi_{i,-4}}{2\left(x-a_{i}\right)^{4}}+\frac{\varphi_{i, 0}}{2}+\widetilde{C}_{1}+\frac{\varphi_{i, 4-l}\left(x-a_{i}\right)^{4-l}}{2}+O\left(\left(x-a_{i}\right)^{5-l}\right),
$$

where $l=1,2,3$. Then $f_{2}^{\prime}(x)=u(x) f_{1}^{\prime}(x)+\frac{u^{\prime}(x) f_{1}(x)}{2}-\frac{f_{1}^{(5)}(x)}{4}$ in a neighborhood of $a_{i}$

$f_{2}=\frac{3\left(\varphi_{i,-4}-280\right) \varphi_{i,-4}}{8\left(x-a_{i}\right)^{8}}+\frac{\varphi_{i,-4}\left(3 \varphi_{i, 0}+2 C_{1}\right)}{4\left(x-a_{i}\right)^{4}}+\frac{3 \varphi_{i,-4} \varphi_{i, 4-l}}{4\left(x-a_{i}\right)^{l}}+O\left(\left(x-a_{i}\right)^{-l+1}\right)$.

We see that the coefficient in the term $\left(x-a_{i}\right)^{-l}$ equals $\varphi_{i, l} \frac{3 \varphi_{i,-4}}{4}$. Consider $f_{k}$

$$
f_{k}=\frac{A_{i,-4 k}^{k}}{\left(x-a_{i}\right)^{4 k}}+\frac{A_{i,-4 k+4}^{k}}{\left(x-a_{i}\right)^{4 k-4}}+\frac{A_{i,-4 k+8-l}^{k}}{\left(x-a_{i}\right)^{4 k-8+l}}+O\left(\left(x-a_{i}\right)^{-4 k+9-l}\right)
$$

Let us show that $A_{i,-4 k+8-l}^{k}=\varphi_{i, l} K_{i,-4 k+8-l}^{k}$, where $K_{i,-4 k+8-l}^{k} \neq 0$ and doesn't depend on $\varphi_{i, 4-l}$. The proof is by induction on $k$. We checked this for $k=2$.

$$
\begin{gathered}
f_{k+1}=\frac{A_{i,-4 k-4}^{k+1}}{\left(x-a_{i}\right)^{4 k+4}}+\frac{A_{i,-4 k}^{k+1}}{\left(x-a_{i}\right)^{4 k}}+\frac{(8 k-4+l) \varphi_{i, 4-l} A_{i,-4 k}^{k}}{2(4 k-4+l)\left(x-a_{i}\right)^{4 k-4+l}}+ \\
+\frac{(4 k-6+l)\left(4 \varphi_{i,-4}-(4 k-8+l)(4 k-7+l)(4 k-5+l)(4 k-4+l)\right) A_{i,-4 k+8-l}^{k}+}{4(4 k-4+l)\left(x-a_{i}\right)^{4 k-4+l}}+ \\
+O\left(\left(x-a_{i}\right)^{-4 k+5-l}\right)
\end{gathered}
$$

So, we have

$$
\begin{gathered}
K_{i,-4 k+4-l}^{k+1}=\frac{(8 k-4+l) A_{i,-4 k}^{k}}{2(4 k-4+l)}+ \\
+\frac{(4 k-6+l)\left(4 \varphi_{i,-4}-(4 k-8+l)(4 k-7+l)(4 k-5+l)(4 k-4+l)\right) K_{i,-4 k+8-l}^{k} .}{4(4 k-4+l)} .
\end{gathered}
$$

We see that if $k \leqslant n_{i}+1$, then $K_{i,-4 k+4-l}^{k+1}>0$. If $k>n_{i}+1$, then from Lemma 3.3 we obtain that $A_{i,-4 k}^{k}=0$. Hence, if $k>n_{i}+1$, then $K_{i,-4 k+8-l}^{k}<0$. But $A_{i,-4 k+8-l}^{k}=\varphi_{i, l} K_{i,-4 k+8-l}^{k}$ and we must find a $g$ such that $f_{g+1} \equiv 0$. It now follows that $A_{i,-4 k+8-l}^{k}=0$ for some $k \Leftrightarrow \varphi_{i, l}=0$.

In general, assume $\varphi_{i, 4 k-l}=0$, where $k=1, \ldots, m-1, l=1,2,3$ and $m \leqslant n_{i}$. 
We have already checked this for $m=2$. Let us prove that $\varphi_{i, 4 m-l}=0$. We have

$$
f_{1}=\frac{\varphi_{i,-4}}{2\left(x-a_{i}\right)^{4}}+\frac{\varphi_{i, 0}}{2}+C_{1}+\sum_{t=1}^{t=m-1} \frac{\varphi_{i, 4 t}\left(x-a_{i}\right)^{4 t}}{2}+\frac{\varphi_{i, 4 m-l}\left(x-a_{i}\right)^{4 m-l}}{2}+\ldots
$$

Then

$$
\begin{aligned}
& f_{k}=\sum_{t=-k}^{t=m-k} A_{i, 4 t}^{k}\left(x-a_{i}\right)^{4 t}+A_{i, 4 m-4 k+4-l}^{k}\left(x-a_{i}\right)^{4 m-4 k+4-l}+\ldots \\
& f_{k+1}=\sum_{t=-k-1}^{t=m-k-1} A_{i, 4 t}^{k+1}\left(x-a_{i}\right)^{4 t}+A_{i, 4 m-4 k-l}^{k+1}\left(x-a_{i}\right)^{4 m-4 k-l}+\ldots
\end{aligned}
$$

Calculations show that

$$
\begin{gathered}
A_{i, 4 m-4 k-l}^{k+1}= \\
=\frac{(4 m-4 k+2-l) A_{i, 4 m-4 k+4-l}^{k}}{4(4 m-4 k-l)} \times \\
\times\left(4 \varphi_{i,-4}-(4 m-4 k+4-l)(4 m-4 k+3-l)(4 m-4 k+1-l)(4 m-4 k-l)\right)+ \\
+\frac{(4 m-8 k-l) \varphi_{i, 4 m-l} A_{i,-4 k}^{k}}{2(4 m-4 k-l)} .
\end{gathered}
$$

If we take $m=1$ in (12), we get (10).

Since $A_{i, 4 m-l}^{1}=\frac{\varphi_{i, 4 m-l}}{2}$, we obtain from (12) that $A_{i, 4 m-4 k-l}^{k+1}=\varphi_{i, 4 m-l} K_{i, 4 m-4 k-l}^{k+1}$, where $K_{i, 4 m-4 k-l}^{k+1}$ doesn't depend on $\varphi_{i, 4 m-l}$.

$$
\begin{gathered}
K_{i, 4 m-4 k-l}^{k+1}= \\
=\frac{(4 m-4 k+2-l) K_{i, 4 m-4 k+4-l}^{k}}{4(4 m-4 k-l)} \times \\
\times\left(4 \varphi_{i,-4}-(4 m-4 k+4-l)(4 m-4 k+3-l)(4 m-4 k+1-l)(4 m-4 k-l)\right)+ \\
+\frac{(4 m-8 k-l) A_{i,-4 k}^{k}}{2(4 m-4 k-l)} .
\end{gathered}
$$

\section{Lemma 3.5}

Number $K_{i, 4 m-4 k-l}^{n_{i}+1} \neq 0$ for $m \leqslant n_{i}$. 
Proof. Consider two cases.

1) $l=2$.

From (13) we obtain that

$$
A_{i,-2}^{m+1}=\frac{(4 m+2) \varphi_{i, 4 m-2} A_{i,-4 m}^{m}}{4}=\varphi_{i, 4 m-2} \frac{(4 m+2) A_{i,-4 m}^{m}}{4}=\varphi_{i, 4 m-l} K_{i,-2}^{m+1} .
$$

We see from Lemma 3.2 that if $m \leqslant n_{i}$, then $K_{i,-2}^{m+1}>0$. But for $k>m$ expression (13) is positive. So $K_{i, 4 m-4 k-2}^{k+1}>0$ for all $k>m$.

2) $l=1$ or $l=3$.

If $K_{i, 4 m-4 n_{i}-l}^{n_{i}+1}=0$, then from (13) we get

$$
\begin{gathered}
\frac{\left(4 m-8 n_{i}-l\right) A_{i,-4 n_{i}}^{n_{i}}}{2\left(4 m-4 n_{i}-l\right)}= \\
=-\frac{\left(4 m-4 n_{i}+2-l\right) A_{i, 4 m-4 n_{i}+4-l}^{n_{i}} \times}{4\left(4 m-4 n_{i}-l\right)} \times \\
\times\left(4 \varphi_{i,-4}-\left(4 m-4 n_{i}+4-l\right)\left(4 m-4 n_{i}+3-l\right)\left(4 m-4 n_{i}+1-l\right)\left(4 m-4 n_{i}-l\right)\right) .
\end{gathered}
$$

But calculating $A_{i, 4 m-4 n_{i}+4-l}^{n_{i}}$ recursively using (12) we see that the expression above is not true.

We know from Lemma 3.3 that $A_{i,-4 k}^{k}=0$ for all $k \geqslant n_{i}+1$. So for $k \geqslant n_{i}+1$ we have

$$
\begin{gathered}
K_{i, 4 m-4 k-l}^{k+1}= \\
=\frac{(4 m-4 k+2-l) K_{i, 4 m-4 k+4-l}^{k}}{4(4 m-4 k-l)} \times \\
\times\left(4 \varphi_{i,-4}-(4 m-4 k+4-l)(4 m-4 k+3-l)(4 m-4 k+1-l)(4 m-4 k-l)\right) \neq 0
\end{gathered}
$$

So, if there exists a $g$ such that $f_{g+1}=0$, then for some $k$ coefficient $A_{i, 4 m-4 k-l}^{k+1}=\varphi_{i, 4 m-l} K_{i, 4 m-4 k-l}^{k}=0 \Leftrightarrow \varphi_{i, 4 m-l}=0$.

Now suppose that $g>n_{i}$. Then

$f_{1}=\frac{\varphi_{i,-4}}{2\left(x-a_{i}\right)^{4}}+\frac{\varphi_{i, 0}}{2}+C_{1}+\sum_{t=1}^{t=m-1} \frac{\varphi_{i, 4 t}\left(x-a_{i}\right)^{4 t}}{2}+\frac{\varphi_{i, 4 m-l}\left(x-a_{i}\right)^{4 m-l}}{2}+\ldots$,

where $m \geqslant n_{i}+1$. We see that

$$
f_{k}=\sum_{t=-k}^{t=m-k} A_{i, 4 t}^{k}\left(x-a_{i}\right)^{4 t}+A_{i, 4 m-4 k+4-l}^{k}\left(x-a_{i}\right)^{4 m-4 k+4-l}+\ldots
$$


If $k>n_{i}$, we get

$$
\begin{gathered}
K_{i, 4 m-4 k-l}^{k+1}= \\
\frac{(4 m-4 k+2-l) K_{i, 4 m-4 k+4-l}^{k}}{4(4 m-4 k-l)} \times \\
\times\left(4 \varphi_{i,-4}-(4 m-4 k+4-l)(4 m-4 k+3-l)(4 m-4 k+1-l)(4 m-4 k-l)\right) .
\end{gathered}
$$

So, we obtain $K_{i, 4 m-4 k-l}^{k+1} \neq 0$ for $l=1,3$ and $k>n_{i}$ because

$\left.4 \varphi_{i,-4}-(4 m-4 k+4-l)(4 m-4 k+3-l)(4 m-4 k+1-l)(4 m-4 k-l)\right) \neq 0$,

If $k=m>n_{i}$ and $l=2$, then $K_{i,-2}^{m+1}=0$ because of factor $(4 m-4 k+2-l)$.

But if $L_{4}$ commutes with an operator of order $4 g+2$, then there exists a $k \leqslant g+1$ such that $A_{i, 4 m-4 k+3}^{k}=A_{i, 4 m-4 k+1}^{k}=0 \Leftrightarrow \varphi_{i, 4 m-1}=\varphi_{i, 4 m-3}=0$.

\section{Theorem 1.1 is proved.}

\subsection{Proof of corollaries and examples}

We know that

$$
f_{2}=\frac{3\left(\varphi_{i,-4}-280\right) \varphi_{i,-4}}{8\left(x-a_{i}\right)^{8}}+\frac{\varphi_{i,-4}\left(3 \varphi_{i, 0}+\widetilde{C}_{1}\right)}{4\left(x-a_{i}\right)^{4}}+\widetilde{C}_{2}+O\left(\left(x-a_{i}\right)\right),
$$

where $\widetilde{C}_{i}$ are constants and depend on $C_{i}$. Also $A_{i,-4\left(n_{i}+1\right)}^{n_{i}+1}=0$ and that's why

$$
f_{n_{i}+1}=\sum_{t=n_{i}}^{t=1} \frac{A_{i,-4 t}^{n_{i}+1}}{\left(x-a_{i}\right)^{4 t}}+\widetilde{C}_{n_{i}+1}+O\left(\left(x-a_{i}\right)\right)
$$

We must choose constants $C_{1}, \ldots C_{g}$ to vanish principal part of Laurent series of function $f_{n_{i}+1}$. This is always possible to do because $A_{i,-4 n_{i}}^{n_{i}+1}$ linearly depend on $C_{1}, A_{i,-4 n_{i}+4}^{n_{i}+1}$ linearly depend on $C_{1}$ and $C_{2}, A_{i,-4 n_{i}+8}^{n_{i}+1}$ depend on $C_{1}, C_{2}, C_{3}, A_{i,-4}^{n_{i}+1}$ depend linearly on $C_{1}, \ldots, C_{n_{i}}$. We know that only constant function can be holomorphic and bounded function on $\mathbb{C}$. Hence $f_{n_{i}+1}^{\prime} \equiv 0$. Corollary 1.2 is proved.

If we take $C_{1}=-42 g_{2}$, then we obtain Example 1.4.

\subsection{Conjecture}

Consider $f_{k}$, where $k \geqslant n_{i}+1$. Assume that $u(x)$ has $m$ poles (in the fundamental parallelogram or $\mathbb{C}$ ). Take $S=\sum_{i=1}^{i=m} n_{i}+1$. If $\varphi_{i, 4 k-l}=0$, 
$\varphi_{i, 4 r-1}=\varphi_{i, 4 r-3}=0$, where $k=0, \ldots, n_{i}$ and $r=n_{i}+1, \ldots, S$. We see from Lemma 3.3 that degree of pole at $a_{i}$ is not greater than $n_{i}$ for any $k$. We get that $A_{i,-4 n_{i}}^{k}$ linearly depends on $C_{1}, \ldots, C_{k-n_{i}}, A_{i,-4 n_{i}+4}^{k}$ linearly depends on $C_{1}, \ldots, C_{k-n_{i}+1}, A_{i,-4}^{k}$ depends on $C_{1}, \ldots, C_{k}$. So, we must choose constants to vanish $S-1$ terms and we have $S$ constants. Apparently this linear system always has solutions, but it is not proved.

\section{Proof of Theorem 1.5}

Consider the differential equation

$$
\frac{d^{n} w(x)}{d x^{n}}+\widetilde{P}_{1}(x) \frac{d^{n-1} w(x)}{d x^{n-1}}+\ldots+\widetilde{P}_{n-1}(x) \frac{d w(x)}{d x}+\widetilde{P}_{n}(x) w(x)=0 .
$$

We know from theory of ordinary differential equations (see [14] chapter 16) that if $w(x)$ has singularity at point $a$, then $P_{i}(x)$ has singularity at point $a$ for some $i$. A singularity is called regular if coefficients $P_{k}$ have pole of order not greater than $k$ for all $k$. Without loss of generality it can be assumed that $a=0$ and we can write (14) in the following form

$$
x^{n} \frac{d^{n} w(x)}{d x^{n}}+x^{n-1} P_{1}(x) \frac{d^{n-1} w(x)}{d x^{n-1}}+\ldots+x P_{n-1}(x) \frac{d w(x)}{d x}+P_{n}(x) w(x)=0,
$$

where coefficients $P_{i}(x)$ haven't pole at point $a$. . Solutions in a neighborhood of regular singularity have the form (see [14] chapter 16)

$$
w(x)=\sum_{m=0}^{\infty} c_{m} x^{m+\sigma} .
$$

By $L$ denote the operator $x^{n} \frac{d^{n} w(x)}{d x^{n}}+x^{n-1} P_{1}(x) \frac{d^{n-1} w(x)}{d x^{n-1}}+\ldots+x P_{n-1}(x) \frac{d w(x)}{d x}+$ $P_{n}(x) w(x)=0$ and $[\sigma+m]_{n}=(\sigma+m)(\sigma+m-1) \ldots(\sigma+n-m+1)$. We get

$$
L w=L\left(\sum_{m=0}^{\infty} c_{m} x^{m+\sigma}\right)=\sum_{m=0}^{\infty} c_{m} x^{m+\sigma} f(x, m+\sigma),
$$

where $f(x, m+\sigma)=[\sigma+m]_{n}+P_{1}(x)[\sigma+m]_{n-1}+[\sigma+m]_{1} P_{n-1}(x)+P_{n}(x)=$ $\sum_{\lambda=0}^{\infty} f_{\lambda}(m+\sigma) x^{\lambda}$. If $L w=0$, then

$c_{0} f_{0}(\sigma)=0$

$c_{1} f_{0}(\sigma+1)+c_{0} f_{1}(\sigma)=0$ 
$c_{m} f_{0}(\sigma+m)+c_{m-1} f_{1}(\sigma+m-1)+\ldots+c_{0} f_{m}(\sigma)=0$.

Since $c_{0} \neq 0$, it follows that

$$
f_{0}(\sigma)=[\sigma]_{n}+[\sigma]_{n-1} P_{1}(0)+\ldots+[\sigma]_{1} P_{n-1}(0)+P_{n}(0)=0 .
$$

Let $\sigma$ be a root of (16). If $f_{0}(\sigma+m) \neq 0$, where $m$ is integer, then we can find constants $c_{m}$ by the following formulas (see [14] chapter 16)

$$
c_{m}=\frac{(-1)^{m} c_{0} F_{m}(\sigma)}{f_{0}(\sigma+1) f_{0}(\sigma+2) \ldots f_{0}(\sigma+m)},
$$

where

$$
F_{m}(\sigma)=\left|\begin{array}{ccccc}
f_{1}(\sigma+m-1), & f_{2}(\sigma+m-2), & \ldots, & f_{m-1}(\sigma+1), & f_{m}(\sigma) \\
f_{0}(\sigma+m-1), & f_{1}(\sigma+m-2), & \ldots, & f_{m-2}(\sigma+1), & f_{m-1}(\sigma) \\
0, & f_{0}(\sigma+m-2), & \ldots, & f_{m-3}(\sigma+1), & f_{m-2}(\sigma) \\
\ldots \ldots & \ldots \ldots & \ldots, & \ldots \ldots ., & \ldots \ldots \\
0, & 0, & \ldots, & f_{0}(\sigma+1), & f_{1}(\sigma)
\end{array}\right|
$$

So, if $\sigma_{i}, \sigma_{j}$ are roots of $f_{0}(\sigma)=0$ and $\sigma_{i}-\sigma_{j}$ is not integer for all $i \neq j$, then all solutions of (14) have the form $x^{\sigma} g(x)$, where $g(x)$ is a holomorphic function. If for some $i, j$ number $\sigma_{i}-\sigma_{j} \in \mathbb{Z}$, then solutions have the form $g_{0}(x) \ln ^{k} x+g_{1}(x) \ln ^{k-1} x+\ldots+g_{k}(x)$, where $g_{i}(x)$ could be equal zero.

Consider the operator $L_{4}=\partial_{x}^{4}+u(x)$. We proved before that if $L_{4}$ commutes with an operator of order $4 g+2$, then $u(x)$ can have pole only of order 4 and $\varphi_{i,-4}=n_{i}\left(4 n_{i}+1\right)\left(4 n_{i}+3\right)\left(4 n_{i}+4\right)$. This means that eigenfunctions of $L_{4}$ have regular singularities. Solutions of (16) have the form

$\sigma_{i, 1}=\frac{1}{2}\left(1-4 n_{i}-\sqrt{1-16 n_{i}-16 n_{i}^{2}}\right)$

$\sigma_{i, 2}=\frac{1}{2}\left(1-4 n_{i}+\sqrt{1-16 n_{i}-16 n_{i}^{2}}\right)$

$\sigma_{i, 3}=\frac{1}{2}\left(5+4 n_{i}-\sqrt{1-16 n_{i}-16 n_{i}^{2}}\right)$

$\sigma_{i, 4}=\frac{1}{2}\left(5+4 n_{i}+\sqrt{1-16 n_{i}-16 n_{i}^{2}}\right)$.

This means that solutions can have logarithmic terms because $\sigma_{i, 3}-\sigma_{i, 1}=$ $\sigma_{i, 4}-\sigma_{i, 2}=4 n_{i}+2$. But we proved that if $L_{4}$ commutes with operator $4 g+2$, then $\varphi_{4 k-l}=0, k=0, \ldots, n_{i}, l=1,2,3$. Hence, eigenfunctions in a neighborhood of pole $a_{i}$ have the form

$\psi_{i, k}(x)=\alpha_{0} x^{\sigma_{i, k}}+\alpha_{4} x^{\sigma_{i, k}+4}+\ldots+\alpha_{4 n_{i}} x^{\sigma_{i, k}+4 n_{i}}+\alpha_{4 n_{i}+1} x^{\sigma_{i, k}+4 n_{i}+1}+\alpha_{4 n_{i}+2} x^{\sigma_{i, k}+4 n_{i}+2}+\ldots$

Easy to check that we can find all coefficients explicitly. So logarithmic terms will not appear. 


\section{References}

[1] Burchnall J.-L., Chaundy T.W. Commutative ordinary differential operators, Proc. London Math. Soc. 21 (1923), 420-440; Proc. Royal Soc. London (A) 118 (1928), 557-583.

[2] I.M. Krichever, Integration of nonlinear equations by the methods of algebraic geometry, Functional Anal. Appl., 11:1 (1977), 12-26.

[3] I.M. Krichever. Commutative rings of ordinary linear differential operators, Functional Anal. Appl., 12:3 (1978), 175-185.

[4] I.M. Krichever, S.P. Novikov. Holomorphic fiberings and nonlinear equations. Finite zone solutions of rank 2, Sov. Math., Dokl. 20(4), 650-654 (1979).

[5] O.I. Mokhov. Commuting ordinary differential operators of rank 3 corresponding to an elliptic curve, Russian Math. Surveys, 37:4 (1982), 129130.

[6] O.I. Mokhov. Commuting differential operators of rank 3, and nonlinear differential equations, Math. USSR, Izvestiya, 35:3 (1990), 629-655.

[7] A.E. Mironov. Self-adjoint commuting ordinary differential operators, Inventiones mathematicae. 2014. Vol. 197, No. 2. P. 417-431.

[8] Dixmier, Jacques. Sur les algebres de Weyl. Bulletin de la Societe Mathematique de France 96 (1968): 209-242.

[9] A.E. Mironov. Periodic and rapid decay rank two self-adjoint commuting differential operators, Amer. Math. Soc. Transl. Ser. 2, V. 234, 2014, P. 309-322.

[10] O. I. Mokhov. On Commutative Subalgebras of the Weyl Algebra Related to Commuting Operators of Arbitrary Rank and Genus, Mathematical Notes, vol. 94:2 (2013), 298-300

[11] O.I.Mokhov. Commuting ordinary differential operators of arbitrary genus and arbitrary rank with polynomial coefficients. Amer. Math. Soc. Transl. Ser. 2, V. 234, 2014, P. 323-336.

[12] V S Oganesyan, "Commuting differential operators of rank 2 and arbitrary genus g with polynomial coefficients", Russian Math. Surveys, 2015, 70 (1), 165-167. 
[13] V.S.Oganesyan. Commuting differential operators of rank 2 with polynomial coefficients, Functional Analysis and Its Applications, 50:1 (2016), arXiv:1409.4058.

[14] E.L.Ince. Ordinary differential equations, Dover, New York, 1956.

[15] F. Grunbaum, Commuting pairs of linear ordinary differential operators of orders four and six. Phys. D, 31:3 (1988), 424-433.

[16] E. Previato, G. Wilson, Differential operators and rank 2 bundles over elliptic curves, Compositio Math. 81:1 (1992), 107-119.

[17] V. N. Davletshina. Commuting differential operators of rank 2 with trigonometric coefficients, Sibirsk. Mat. Zh, Volume 56 (2015), 513-519.

[18] V. N. Davletshina. On self-adjoint commuting differential operators of rank two, Sib. Èlektron. Mat. Izv, Volume 10 (2013), 109-112. 